\title{
Síndrome de Down, cerebro y desarrollo
}

\section{Down syndrome, brain and development}

\author{
Andrés Molero Chamizo ${ }^{1}$ \\ Universidad de Huelva, España \\ Guadalupe Nathzidy Rivera Urbina \\ Universidad Pablo de Olavide, Sevilla, España
}

(Rec: julio de 2012 - Acep: julio de 2012)

\begin{abstract}
Resumen
En esta revisión describiremos brevemente los aspectos fundamentales que caracterizan a la Psicología del Desarrollo y a la Neurociencia, como disciplinas científicas necesarias en el estudio y la comprensión del desarrollo ontogenético y sus trastornos. Esto nos permitirá concretar la naturaleza de las distintas etapas del desarrollo, y evaluar los sustratos cerebrales de la conducta asociados a estos cambios evolutivos. Finalmente, expondremos las características neurobiológicas y evolutivas de un trastorno del neurodesarrollo determinado genéticamente, el síndrome de Down.

Palabras clave: cerebro, desarrollo, síndrome de Down, trastorno y trisomía.
\end{abstract}

\begin{abstract}
In this review we briefly describe the main aspects that characterize the Developmental Psychology and Neuroscience as scientific disciplines necessary in the study and understanding of ontogenetic development and it disorders. This allows us to specify the nature of the different stages of development and to evaluate the neural substrates of behavior associated with these evolutionary changes. Finally, we discuss the neurobiological and evolutionary characteristics of a genetically determined neurodevelopmental disorder, the Down syndrome.

Keywords: brain, development, disorder, Down syndrome and trisomy.
\end{abstract}

\footnotetext{
1 Dirección para correspondencia (Correspondence address): Andrés Molero Chamizo. Universidad de Huelva. Área de Psicobiología. Avda. Fuerzas Armadas. 21071. Campus El Carmen. Email: andres.molero@dpsi.uhu.es. Teléfono. (34)959219503. Fax: (34)959219201.
} 


\section{Introducción}

Uno de los aspectos más relevantes para la Psicología, es conocer los procesos y los mecanismos psicológicos comunes que dirigen nuestro desarrollo personal. La Psicología del desarrollo estudia estos procesos para conocer las leyes y los principios generales que gobiernan nuestra conducta en las distintas etapas del desarrollo individual u ontogenético. De este modo se puede prever y anticipar los patrones conductuales y cognitivos característicos de las distintas edades por las que transcurre el ser humano. Así, por ejemplo, la Psicología puede intervenir en cada etapa evolutiva para reforzar, potenciar o mejorar las capacidades, habilidades y disposiciones individuales. Por otra parte, conocer los procesos psicológicos característicos de cada etapa, puede ayudar también, en aquellos casos en los que se identifiquen ciertas deficiencias, retrasos o trastornos. Esta posibilidad resulta especialmente cierta en multitud de contextos educativos. En este sentido, la Psicología de la Educación, otra área de la Psicología, dirige fundamentalmente su atención a estos procesos evolutivos que tienen lugar en el ámbito de la educación.

Como complemento necesario al conocimiento de las características de los estadios de nuestra vida, una rama de la Ciencia, denominada Neurociencia, intenta comprender, además, cuáles son los mecanismos biológicos que subyacen a los distintos procesos conductuales, cognitivos, emocionales, e incluso motivacionales, que acompañan a cada etapa evolutiva (Kandel et al., 2003). La experiencia alcanzada hoy día desde muchas áreas de la Neurociencia, en particular desde la Psicobiología del desarrollo, así como las notables aportaciones derivadas de los avances tecnológicos, especialmente las técnicas de imagen cerebral o neuroimagen, están permitiendo conocer los determinantes neurobiológicos del desarrollo ontogenético (Stiles \& Jernigan, 2010), así como la etiopatogenia de muchos trastornos evolutivos (Harvey \& Boksa, 2012; NicklJockschat \& Michel 2011).

Todos estos aspectos fundamentales de nuestras etapas de desarrollo y de los sustratos neurales subyacentes serán tratados en esta revisión. En la primera parte se darán a conocer cuáles son esos principios psicológicos descritos a lo largo del ciclo vital por la Psicología del desarrollo. Puesto que estos principios necesitan igualmente ser explicados por mecanismos biológicos, se completará este primer apartado con una descripción breve de los aspectos conocidos de la neurobiología del desarrollo ontogenético. Finalmente, en la tercera parte pondremos estos conocimientos de la Neurociencia en relación con uno de los trastornos del desarrollo con mayor relevancia clínica, el síndrome de Down.

\section{Psicología del desarrollo}

Numerosos investigadores han aportado sus experiencias y sus estudios para intentar definir cuáles son las características psicológicas de los sucesivos estadios vitales del desarrollo. Algunos ejemplos representativos pueden encontrarse en las figuras de Jean Piaget (1896-1980) y Lev Semionovich Vygotsky (1896-1934). Del primero destacan obras como La naissance de l'intelligence chez l'enfant (1977) (El nacimiento de la inteligencia en el niño, 1985, en la traducción española), o La psychologie de l'enfant (1969) (Psicología del niño, 1993, en la traducción española). En cuanto a Vygotsky, éste dedicó gran parte de su corta, pero intensa labor investigadora y productiva, a la Psicolingüística, es decir, a tratar de entender los complejos mecanismos psicológicos vinculados al lenguaje. Sus teorías e hipótesis tienen, aun hoy día, una gran importancia a la hora de explicar los procesos de aprendizaje que emergen durante el desarrollo. Existe una traducción española de su gran obra Pensamiento y lenguaje, que fue escrita en el año 1934, en la que el propio Piaget interviene aportando algunos comentarios críticos a la obra (Vygotsky, 1993).

La Psicología del desarrollo está interesada en todos los procesos que se suceden a lo largo del ciclo vital, desde el nacimiento hasta la senectud. Sin embargo, es cierto que el estudio de las primeras etapas de desarrollo, sobre todo la infancia y la adolescencia, han sido el principal objeto de atención para los investigadores. Por este motivo, aquí se comentarán brevemente sólo algunos de los principios y procesos descritos específicamente en estas particulares etapas evolutivas. Para tal propósito tomaremos como referencia las aportaciones de J. Piaget y L. S. Vygotsky, dos de los grandes teóricos del desarrollo.

Piaget nació en Suiza en 1896 (el mismo año en que también nació Vygotsky). Centró su interés en identificar los procesos psicológicos característicos de las distintas etapas de crecimiento del niño. Sus investigaciones le llevaron a describir una serie de estadios evolutivos, que son ya conceptos clásicos en el campo de la Psicología del Desarrollo. Los procesos que identificó fueron más allá del plano puramente psíquico, y elaboró un complejo tránsito evolutivo que incluía tanto respuestas sensorio-motoras, como el desarrollo de las percepciones. No obstante, las 
aportaciones más relevantes para el propósito de este trabajo, y para la Psicología Evolutiva o del Desarrollo, fueron aquellas que derivaron de sus explicaciones acerca de las funciones semióticas y simbólicas que desarrolla el niño en determinados estadios, así como de las operaciones concretas del pensamiento que el niño y el adolescente, son capaces de mostrar en los distintos momentos evolutivos.

Piaget consideraba los periodos previos a la adquisición del lenguaje como un nivel evolutivo sensorio-motor. En su teoría del desarrollo, el lactante, previamente a la formación del lenguaje, ya muestra un grado de inteligencia con un componente práctico. Una inteligencia dirigida a satisfacer determinadas demandas y a resolver problemas esencialmente de acción (Piaget, 1977, 1985). La característica de estas etapas es la ausencia de funciones simbólicas, como ocurre en el lenguaje. No obstante, según Piaget, el niño aquí elabora unas construcciones apoyadas exclusivamente en respuestas sensoriales y motoras. En este nivel sensorio-motor, Piaget propuso la continuidad de hasta cinco estadios bien diferenciados (Piaget \& Inhelder, 1969, 1993). En un sexto estadio, pasados los doce primeros meses, el niño prácticamente se encuentra resuelto de su periodo netamente sensorial y motor, y transita a un nuevo periodo en el que ya no se encuentra permanentemente sujeto a la percepción, sino que es capaz de interiorizar y comprender determinadas situaciones. No obstante, el niño no supera por completo los niveles previos en este otro nivel. Más bien acumula sus experiencias. Y a partir de este momento, en el nivel senso-motor se consigue el inicio de una construcción de lo real, que incluye, según Piaget, aspectos cognoscitivos y afectivos vinculados a las reacciones sensoriales y motoras. Entre este primer gran periodo de desarrollo y las posteriores etapas simbólicas, de operaciones y de representaciones, Piaget propone un segundo gran periodo relacionado esencialmente con las percepciones. Las estructuras senso-motoras descritas y su evolución perceptual, constituyen, para este autor, un paso necesario hasta alcanzar el momento en el que son posibles las operaciones del pensamiento.

Piaget entiende que en torno al año y medio, o dos años, el niño comienza a elaborar representaciones en el pensamiento. Tras la evolución de los procesos sensoriales, motores y perceptivos, el niño alcanza un estadio donde por primera vez surgen funciones semióticas y simbólicas. En este tercer gran momento del desarrollo cognitivo, el niño pude representar estímulos no presentes o perceptibles. Piaget enumeró una serie de sucesos de la infancia, donde se hacía evidente la construcción de significantes diferenciados para referirse a estos estímulos o situaciones no presentes: la imitación diferida, el juego simbólico, el dibujo, la formación de imágenes mentales, y, por último, la adquisición del lenguaje (Piaget \& Inhelder, 1993). Estas funciones semióticas prevalecen durante años, y no es, sino a la edad de siete $u$ ocho años, cuando pueden identificarse los primeros procesos de interiorización directa de las acciones en operaciones del pensamiento. En este momento (cuarto periodo de desarrollo en la teoría piagetiana, y que se extiende hasta la edad de los once o doce años), se suceden tres niveles de tránsito de la acción, la cual ha predominado hasta ahora, sobre la verdadera operación. Durante este periodo se completan las operaciones concretas del pensamiento y se definen las relaciones interindividuales. Pero no se trata de una etapa definitiva para el desarrollo del pensamiento. Este periodo evolutivo habrá de ser perfeccionado respecto a la naturaleza de las construcciones que en él tienen lugar, puesto que, según Piaget, son construcciones aún limitadas y con necesidades propias de las operaciones concretas. Asimismo, la evolución afectiva, relacional y moral del niño, permanece necesariamente ligada a los cambios cognitivos característicos de este gran periodo de desarrollo.

En términos generales, la teoría de Piaget conduce a un último gran periodo de desarrollo psicológico del niño, que transcurre desde los once-doce años hasta los catorce o quince (Piaget \& Inhelder, 1993). Aquí es cuando, esencialmente, el individuo se libera de lo concreto y desarrolla las habilidades necesarias para transformar la realidad percibida. Las transformaciones del pensamiento que se alcanzan en este quinto periodo de desarrollo, permiten la proliferación de hipótesis y razonamientos desligados de lo concreto y de lo que está presente o es actual. Así, la formación de esquemas, leyes y pensamientos formales constituyen el conjunto de las operaciones formales asociadas a este periodo de desarrollo. Como bien sabe la Neurociencia hoy día, todas estas posibilidades de razonamiento, abstracción y de juicios morales descritas en la teoría piagetiana, y en otros modelos de desarrollo, están biológicamente ligadas a la maduración de un área de corteza cerebral especializada en la convergencia de tales elaboraciones del pensamiento: la corteza prefrontal (Goldberg, 2004).

Las aportaciones de Piaget aquí descritas son una referencia en el estudio de la Psicología del desarro1lo. Sus planteamientos genéricos fueron, a su vez, un preciado argumento intelectual para que otros autores importantes extrajesen de su obra nuevas teorías y 
conceptos válidos para el estudio de la conciencia y el pensamiento. Es el caso de la producción científica de Vygotsky, que, por otra parte, fue mucho más valorada y reconocida varios años después de su publicación y difusión. Vygotsky nació en la antigua U.R.S.S. y fue coetáneo de Piaget. Estudió críticamente los argumentos del investigador suizo y enlazó las piezas necesarias de su teoría del desarrollo para descubrir experimentalmente las intrigantes relaciones entre pensamiento y lenguaje. Expondremos algunas de sus explicaciones acerca de los mecanismos psicológicos del lenguaje, con el propósito de entender qué procesos evolucionan en nuestro pensamiento desde la infancia, y cómo lo hacen.

En realidad, la divergencia entre Vygotsky y las teorías existentes hasta entonces sobre la relación del lenguaje con el pensamiento, derivaba, sencillamente, de los métodos de análisis para estudiar ambas estructuras psicológicas. Para el autor soviético era un error analizar el lenguaje y el pensamiento como elementos independientes, aislados o separados. El pensamiento y la palabra están inevitablemente en continua interacción como un proceso unitario. De lo contrario, el componente semántico del lenguaje (el significado), habría de ser estudiado, únicamente, como un proceso de pensamiento que varía y se modifica independientemente de los fonemas o las palabras que lo representen. Y, por el mismo motivo, las palabras deberían ser consideradas tan sólo como sonidos. Vygotsky también criticó esta separación entre los aspectos fonéticos y semánticos que imperaba en el estudio del desarrollo del lenguaje en el campo de la Psicología Infantil. En opinión de este autor, el desarrollo lingüístico no puede ser analizado correctamente atendiendo a la evolución fonética o al desarrollo del pensamiento por separado. La mejor forma de comprender la naturaleza de la relación entre lenguaje y pensamiento es, en palabras de Vygotsky, una aproximación "por unidades", entendido esto como un estudio de las propiedades totales de la relación de ambos, de modo que no puedan ser escindidos ninguno de sus componentes sin que pierda sentido la totalidad (Vygotsky, 1993). Esta concepción unitaria del desarrollo cognitivo, aparentemente manida en otros ámbitos de la Psicología, ha ido reforzándose con el tiempo hasta calar profundamente en la Psicolingüística y la Psicología del Desarrollo. La aportación más relevante, quizá, para entender la evolución de los procesos cognitivos, y, por tanto, para el propósito de esta revisión, puede hallarse en su explicación de la unidad de pensamiento verbal que no puede ser dividida ni separada del pensamiento y el lenguaje. Y, para Vygotsky, esa unidad es el propio significado de las palabras. En el significado se unen el pensamiento y el lenguaje para formar un pensamiento verbal, dado que el significado en sí de una palabra es pensamiento y es habla (Vygotsky, 1993). La conclusión inmediata que deriva de esta interpretación es que para entender el lenguaje como un proceso de pensamiento, habrá que descubrir primero cuáles son los mecanismos responsables de otorgar significado a las palabras. Esta concepción implica la posibilidad de analizar el lenguaje y el pensamiento como un todo unitario, mediante el conocimiento de tales mecanismos.

No obstante, todo lo anterior no hace sino determinar el objeto de estudio (la semántica) para comprender cómo nuestros procesos mentales se relacionan con el lenguaje, pero no explica aún, cuáles son los mecanismos que subyacen a la formación de significados por medio de las palabras. En la infancia, de acuerdo a la interpretación más elemental de las teorías de Vygotsky, no se pueden negar los procesos de comprensión y comunicación. Sin embargo, y aquí tal vez reside la explicación clave para la Psicología Evolutiva y la Psicolingüística, no es sino en el desarrollo avanzado del significado de las palabras, cuando se puede alcanzar una comunicación real. Y este desarrollo significa que el individuo hace suyos aquellos conceptos que están construidos como ideas generalizadas. Dicho de otra manera, sólo cuando se comparten significados generalizados se puede conseguir una verdadera comprensión. La falta de significado, de concepto, asociado a una palabra deja a ésta simplemente en el mundo de los sonidos. Una palabra comunicante sólo lo es cuando significa un concepto convencionalmente generalizado. Así, el niño puede desarrollar una comunicación por medio del lenguaje, en un proceso de maduración e interiorización de conceptos relacionados con palabras. Cuando un niño ha adquirido un concepto, en ese momento puede encontrar la palabra para significarlo. $\mathrm{Y}$ en etapas posteriores del desarrollo, la relación entre lenguaje y pensamiento se hace aún más compleja. Para Vygotsky, el significado de una palabra alcanza a ser un pensamiento generalizado, y es de este modo cómo las palabras consiguen comunicar ideas establecidas por convención (Vygotsky, 1993). Pero este pensamiento en sí posee además un componente social $\mathrm{y}$, sobre todo, emocional, proyectado sobre la realidad a la que se refiere. Esta compleja unidad formada por palabra, pensamiento y afectos se alcanza cuando el niño encuentra un significado, por medio del lenguaje, de la realidad que le rodea. 
En definitiva, las teorías de Vygotsky sobre pensamiento y lenguaje han servido para analizar y tratar de entender cómo evolucionan los elaborados procesos psicológicos del ser humano. Y esta evolución puede considerarse uno de los grandes objetivos de estudio de la Psicología del Desarrollo. En el siguiente apartado se hará una breve descripción del desarrollo del cerebro, al menos en sus aspectos más relevantes. El objetivo es conocer algunos de los sustratos neurobiológicos que subyacen al desarrollo de las capacidades cognitivas y afectivas del niño.

\section{Desarrollo del cerebro}

A principios del siglo XX, Santiago Ramón y Cajal supo intuir las características citoarquitectónicas y funcionales del sistema nervioso de los vertebrados. Sus descripciones detalladas acerca de la distribución celular del sistema nervioso y de sus características han podido ser confirmadas con el paso del tiempo (Ramon y Cajal 1904), gracias a la disponibilidad de una tecnología muy sofisticada y efectiva. Sus aportaciones permitieron orientar la investigación sobre la anatomía y el desarrollo del sistema nervioso hasta nuestros actuales conocimientos. En este apartado se describirán algunas de las etapas esenciales en el origen y el crecimiento del sistema nervioso central que deben conocerse para entender, por un lado, el normal desarrollo ontogenético y, por otro, las diversas anomalías resultantes de algún defecto en su formación.

En el desarrollo ontogenético del sistema nervioso resulta crucial la tercera semana de gestación (Diamond, Scheibel \& Elson, 1997; Preatoni, 2003; Sanes, Reh \& Harris, 2002). Tras 23 días desde la concepción, el blastodermo, o tejido embrionario, dará lugar a la formación de una estructura denominada placa neural, desde la cual, a su vez, se desarrollará el cerebro y la médula espinal, en un proceso denominado neurulación (Green \& Copp, 2009). En torno a este periodo, la placa neural adopta una forma plegada, y un surco va recorriendo este tejido originando la aparición de una cavidad conocida como tubo neural (De Marco, Merello, Cama, Kibar \& Capra, 2011). Al tiempo que se produce la fusión de los pliegues de la placa, algunas células embrionarias se separan y conforman un tejido conocido como cresta neural. La diferenciación de las células de la cresta neural dará lugar a la formación del ganglio de la raíz dorsal de la médula espinal, a los ganglios sensitivos de los nervios craneales, a la cadena ganglionar simpática del sistema nervioso autónomo, a células gliales de Schwann y a las células de la médula de las glándulas suprarrenales, entre otras estructuras y grupos celulares.

Del resto de la placa neural derivarán el cerebro, o encéfalo, y la médula espinal (Kalat, 2004; Puelles, Martínez \& Martínez, 2008). El cierre del tubo neural dará lugar a la formación de tres vesículas diferenciadas, que en dirección rostral-caudal son: la vesícula del cerebro anterior, la vesícula mesencefálica y la vesícula del cerebro posterior (Copp, 2005). Estas vesículas conformarán, antes del nacimiento, las divisiones anatómicas del prosencéfalo, mesencéfalo y rombencéfalo, respectivamente (Carlson, 2010; Del abril et al., 2001). El prosencéfalo incluirá, asimismo, otras dos subdivisiones anatómicas: el telencéfalo y el diencéfalo. El desarrollo de la primera de estas dos subdivisiones finalizará con la formación de la corteza cerebral, los ventrículos laterales, los ganglios de la base y el sistema límbico. El desarrollo del diencéfalo concluirá con la formación del tercer ventrículo, el tálamo, el hipotálamo y el subtálamo. Por su parte, el mesencéfalo lo formarán dos estructuras bien diferenciadas: el tectum y el tegmentum, constituidas a su vez por varios núcleos celulares. El acueducto cerebral es la estructura del sistema ventricular que formará parte de la anatomía del mesencéfalo. Por último, en el desarrollo del rombencéfalo, podrán diferenciarse otras dos subdivisiones: el metencéfalo y el mielencéfalo. La primera de ellas estará conformada por la protuberancia, el cerebelo y el cuarto ventrículo. Del mielencéfalo derivará una única estructura, el bulbo raquídeo (o médula oblongada).

Por otra parte, las células del extremo caudal del tubo neural, denominadas células de la matriz, madurarán hasta formar, en torno a la tercera semana de desarrollo, los primeros neuroblastos (células primarias neuronales) y glioblastos (células primarias gliales) de la futura médula espinal (Sadler, 2005; Snell, 1999). A partir de la quinta semana, aproximadamente, estos neuroblastos se agruparán en dos regiones bilaterales, conocidas como placa alar y basal, que darán lugar, respectivamente, a las neuronas sensitivas y motoras de la sustancia gris de la médula espinal. Alrededor de la semana 28 ya puede identificarse el asta posterior $y$ anterior de la sustancia gris de la médula espinal, así como la anatomía de la sustancia blanca (Segovia \& Guillamón, 1988). Todos estos procesos de desarrollo, y su normal evolución, están fuertemente determinados por factores genéticos (Parellada, 2001; Pinos Sánchez, Collado Guirao \& Sánchez-Santed, 2005; Plomin, 2002; Stansfield, 1992), pero existen múltiples influencias no genéticas, o epigenéticas, que condicionan la 
formación del cerebro y la médula espinal (Hsiao \& Patterson, 2012; Kandel, Jessell \& Schwartz, 2003; Nelson, 1996; Pinel, 2001). Las etapas en las que toda influencia, genética o epigenética, es determinante se denominan etapas críticas del desarrollo. En cualquiera de estas etapas pueden producirse errores o fallos que tendrán una consecuencia drástica en el curso evolutivo del sistema nervioso y en las conductas que éste controla (Farel, 2003; Gat-Yablonski, 2011; Galaburda \& Cestnick, 2003). Algunos de las alteraciones congénitas producidas por anomalías del desarrollo son la hidrocefalia, la anencefalia, el cretinismo y la espina bífida (Copp \& Greene, 2010). Otros trastornos frecuentes que también parecen tener un origen, al menos en parte, en alguna anomalía de las etapas críticas del desarrollo son la dislexia, el trastorno por déficit de atención con o sin hiperactividad, el autismo y el síndrome de Down. Este último tiene un claro componente genético, y dada la notable prevalencia de casos y su importancia en la Psicología Evolutiva, dedicaremos el siguiente apartado a describir sus características y algunos de los aspectos conocidos de su etiología y neuropatología.

\section{Sindrome de Down}

El progresivo desarrollo a través del tiempode las disciplinas de la anatomía, la fisiología y la bioquímica del cerebro, está permitiendo dar respuesta a la interrogante de cuáles son los mecanismos de un gran abanico a de funciones cerebrales complejas. Los conocimientos disponibles son limitados, y están sujetos al avance tecnológico, pero, con todo, en la actualidad se están dilucidando parte de los sustratos neurobiológicos del lenguaje, la atención, la memoria, los procesos perceptivos, e incluso de la imaginación, la creatividad, la inteligencia y la sexualidad (Changeux, 1994; Hernández-Muela, Mulas \& Mattos, 2004; Le vay, 1995; Rosenzweig \& Leiman, 2002). Conocer cómo se elaboran nuestras emociones, cogniciones, motivaciones y comportamientos es, a su vez, una condición necesaria para descubrir qué mecanismos neuropatológicos son responsables de las diversas alteraciones neurológicas, neuropsicológicas y psiquiátricas existentes. Las diversas ramas de la Neurociencia nos están mostrando la neurobiología de varios de estos trastornos, de los cuales, hasta no hace mucho, tan sólo se tenía conocimiento desde la experiencia clínica. Los trastornos neurodegenerativos (Beal, 2000) y del desarrollo (Mardomingo, 1997) son claros ejemplos de anomalías cuya terapia puede verse enormemente beneficiada por el conocimiento de la neurofisiología, neurofarmacología y neuropatología cerebral. Uno de estos trastornos del desarrollo, que limita la capacidad cognitiva, además de provocar múltiples complicaciones médicas, es el síndrome de Down. Su componente genético y sus efectos sobre el desarrollo cerebral, cognitivo y físico, pueden servir para ejemplificar el modo en el que los factores genéticos y epigenéticos comprometen el normal desarrollo del sistema nervioso y del individuo. Así pues, dedicaremos el resto de este apartado a describir las características clínicas y la etiopatogenia conocida del síndrome de Down. Asimismo, debido a la relación anatomopatológica que existe entre este síndrome y la demencia degenerativa primaria tipo Alzheimer, se analizarán brevemente algunos mecanismos cerebrales comunes que pueden ayudar a entender la naturaleza de las limitaciones funcionales de las personas con síndrome de Down.

Son varias las formas de mutaciones genéticas que afectan al desarrollo del sistema nervioso al incidir sobre la síntesis proteica. Una de ellas consiste en un defecto genético que resulta en la formación de células con un cromosoma de más en alguno de los 23 pares, es decir, en la formación de un individuo con 47 cromosomas. A esta anómala condición, en la que en uno de los pares cromosómicos de las células se observan tres cromosomas, se le denomina trisomía. Una trisomía es una de las formas de aneuploidía celular. Aneuploidía es el término empleado para definir una condición celular en la que existe un número de cromosomas que no es múltiplo exacto de la condición haploide. A su vez, la condición haploide es aquella en la que la célula presenta el número reducido de cromosomas. En la especie humana, las células haploides presentan 23 cromosomas. Los gametos en la meiosis son haploides, toda vez que en la división celular contienen el número reducido de cromosomas (la mitad, es decir, 23). Así pues, una trisomía es una forma de aneuploidía, en la que el número total de cromosomas (47) no es múltiplo de la condición haploide (23). En cuanto al síndrome de Down, puede decirse que está causado por una aneuploidía en forma de trisomía del par cromosómico 21 (Patterson, 2009). El porcentaje más alto de casos de síndrome de Down se debe a una trisomía plena del par cromosómico 21 (Gardiner, Herault, Lott, Antonarakis, Reeves, \& Dierssen, 2010). El resto de casos se debe a mutaciones en las que uno de los cromosomas del par 22 contiene una parte de uno de los cromosomas 21, la cual se ha anexado durante la meiosis o división celular (Gardiner et al., 2010). Esta colocación anómala de un cromosoma -o parte de él- en otro, se conoce como translocación. Las causas de estas alteraciones 
genéticas no se han identificado de manera consistente, pero se piensa que varios factores de riesgo pueden converger para forzar este tipo de mutación (Coppedè, 2009; Scott, Holding, Purcell, Tutty \& Lindow, 2009).

Un porcentaje menor de casos de trisomía 21 se debe a una doble copia procedente del padre, debido a un error en la división de los gametos o células reproductoras. Pero en la mayor parte de los casos la doble copia procede de un error en la división celular de la madre. En ambas condiciones el error suele consistir en una no disyunción, o no separación, de los cromosomas del par 21 en la anafase, o primera fase, de la meiosis; la cual ocurre con mayor incidencia en los autosomas (o cromosomas no sexuales) del par 21 que en el resto de los autosomas (Gardiner et al., 2010).

La trisomía del par cromosómico 21 condiciona drásticamente el normal desarrollo del sistema nervioso (Kleschevnikov, Belichenko, Salehi \& $\mathrm{Wu}, 2012)$ y del resto del organismo. Son abundantes las anomalías o manifestaciones clínicas que derivan de este fallo genético: pliegue del epicanto (pliegue de la piel que cubre el ángulo interno del ojo), corta estatura, dermatoglifia anormal (alteración en las eminencias superficiales, o huellas, de la piel de las manos y pies), hipotonía, microcefalia, y retraso mental y disfunción cognitiva. Desde el punto de vista cognitivo la más relevante es el retraso mental que deriva, en mayor o menor medida, de la afectación cerebral durante etapas tempranas del desarrollo. En lo que sigue describiremos brevemente cómo la génesis de un cromosoma 21 añadido consigue alterar el normal desarrollo del cerebro y de sus funciones, y cómo el resultado de esta mutación induce una neuropatología algo similar a la que causa la demencia tipo Alzheimer.

Las investigaciones genéticas han hallado que la derivación en tres cromosomas 21 por fallo en la meiosis de cualquiera de los progenitores, origina en el individuo con trisomía una desregulación de la expresión génica que afecta al desarrollo prenatal del cerebro (Patterson, 2009; Sommer \& Henrique-Silva, 2008). Una de las consecuencias neurológicas derivadas de la sobre-expresión génica por trisomía 21, es la contrastada afectación de los procesos de diferenciación y maduración cerebrales. La expresión anómala de genes provocada por dicha trisomía afecta particularmente a la diferenciación y especialización celular prenatales, y se asocia con una maduración y desarrollo cerebral retardados, con displasia cortical, con un menor número de neuronas y con procesos de sinaptogénesis reducidos (Gardiner, 2003). Los cambios que se producen en el sistema nervioso central (y sus consecuencias funcionales) se hacen más evidentes después del nacimiento. Posteriormente, las alteraciones se acentúan al final de la infancia y comienzos de la niñez, y son especialmente prominentes en la adolescencia.

Los avances en los últimos lustros han permitido identificar diversos procesos neuropatológicos relacionados con las alteraciones del neurodesarrollo, y con el retraso mental subsiguiente, en el síndrome de Down (Wiseman, Alford, Tybulewicz \& Fisher, 2009). Esta neuropatología se ha identificado en forma de atrofia de la corteza temporal y frontal (particularmente de la corteza prefrontal dorsolateral y orbitofrontal), menor densidad de células granulares corticales, menor volumen de la formación hipocampal y ganglios de la base y cerebelo, déficit en la mielinización de fibras corticales asociativas, menor densidad sináptica en el área 17 de Brodmann (corteza visual primaria) y en los núcleos arqueado y ventromedial del hipotálamo, y una menor producción hipotalámica de factor liberador de hormona del crecimiento $(\mathrm{GH})$ (Dierssen, Herault \& Estivill, 2009). En niños con este síndrome también se han observado unas anomalías características en las dendritas de neuronas piramidales, las cuales muestran espinas dendríticas mal desarrolladas e ineficaces para la neurotransmisión (Garner \& Wetmore, 2012).

Estas alteraciones del neurodesarrollo limitan en grado variable el desempeño cognitivo y las funciones neuropsicológicas (Bartesaghi, Guidi \& Ciani, 2011). En este sentido, la afectación hipocampal compromete la memoria explícita. La alteración de los lóbulos frontales y temporales afecta al aprendizaje, la memoria, la adquisición del lenguaje y las funciones ejecutivas (Lott \& Dierssen, 2010). El menor desarrollo de los ganglios de la base incide sobre la adquisición de destrezas motoras y sobre el control del movimiento. La alteración del cerebelo afecta a la coordinación motora $\mathrm{y}$ al condicionamiento palpebral (un tipo de aprendizaje en el que un estímulo neutro asociado a un soplo en el párpado induce posteriormente una respuesta condicionada de parpadeo).

Como se mencionó anteriormente, la relación entre síndrome de Down y demencia tipo Alzheimer, es de especial importancia para entender la neuropatología de este trastorno del desarrollo y cómo sus consecuencias funcionales pueden derivar en diferentes grados de limitación cognitiva. Por lo tanto, el conocimiento de los aspectos neurobiológicos comunes entre ambas patologías puede ayudar al propósito de esta revisión. 
En la temprana adultez de las personas con síndrome de Down existe una marcada mayor incidencia de este tipo de demencia degenerativa con respecto al resto de la población (Parajuá-Pozo \& Casis-Arguea, 2000). Además, en los sujetos con síndrome de Down se observa la neuropatología característica de la demencia de Alzheimer a partir de los treinta y cinco años, o incluso antes (Perluigi \& Butterfield, 2012). En esta enfermedad, la formación de placas seniles (acumulaciones extracelulares de proteína $\beta$-amiloide alterada) y ovillos neurofibrilares (acumulación anómala de proteína tau intracelular que ataca los microtúbulos de las neuronas) provoca una neurodegeneración progresiva que causa los síntomas de la demencia (Molero et al., 2007). Los sujetos con síndrome de Down que alcanzan esta edad ya muestran concentraciones de $\beta$-amiloide elevadas (en proporciones similares a las de los pacientes con Alzheimer, y en mucha mayor proporción que en los ancianos que no muestran signos de esta enfermedad). Estas concentraciones de proteína amiloide anómala aumentan progresivamente hasta los cincuenta años (Fillat, Dierssen, de Lagrán, \& Altafaj, 2010). La formación de ovillos neurofibrilares (que son un signo patognómico, o característico, de la demencia de Alzheimer) es menos frecuente antes de los treinta años en el síndrome de Down, aunque a partir de los cincuenta años esta neuropatología también se hace evidente (Shi, Kirwan, Smith, MacLean, Orkin $\&$ Livesey, 2012). Una gran proporción de adultos con síndrome de Down presenta demencia, pero el número de casos con este trastorno es algo menor de lo que cabría esperar teniendo en cuenta que la práctica totalidad de ellos presenta la neuropatología del Alzheimer. Las investigaciones actuales están tratando de explicar por qué no todos los sujetos con la neuropatología del Alzheimer y síndrome de Down, manifiestan signos de demencia tipo Alzheimer (Fillat et al., 2010).

La clave de esta disonancia parece estar en el tipo de proteína alterada que se acumula en ambos casos, y el posible daño cerebral subsecuente. En el caso de la demencia de Alzheimer, la proteína alterada que forma las placas seniles, o neuríticas, procede de otra proteína denominada apolipoproteína. El gen que codifica esta apolipoproteína está situado en el par cromosómico 21. Una mutación en este gen parece estar relacionada con la formación de las placas neuríticas características de la enfermedad de Alzheimer (Bertram \& Tanzi, 2012). Una de las formas alélicas del gen de la apolipoproteína que probabiliza esta enfermedad es el alelo APO E4, el cual produce un tipo de apolipoproteína conocido como APO E. Poseer el alelo de la APO E supone un factor de riesgo crítico que predispone a la demencia en la adultez (Frieden \& Garai, 2012). En el caso del síndrome de Down, existe un mayor riesgo de acumular $\beta$-amiloide derivada de la expresión de este alelo (Sabbagh, Fleisher, Chen, Rogers, Berk, Reiman et al., 2011), entre otras razones debido a que las células de estos individuos presentan genes en el par 21 por triplicado. El tipo de mutación genética es clave, pues, para entender por qué algunos casos de síndrome de Down con neuropatología característica del Alzheimer no desarrollan demencia (Fillat et al., 2010).

Algunas investigaciones han mostrado que menos del 50\% de los individuos con síndrome de Down expresa signos de demencia (Nieuwenhuis-Mark, 2009). En aquellos casos en los que se observa demencia, ésta suele aparecer en torno a los cincuenta años de edad, bastante tiempo después de que se haya iniciado en ellos la neuropatología del Alzheimer. Por otra parte, algunos estudios indican que la atrofia cerebral y la dilatación de los ventrículos características del Alzheimer suele observarse en el síndrome de Down con demencia, pero no en aquellos casos en los que no se evidencian signos de demencia (Parajuá-Pozo \& Casis-Arguea, 2000). La neuropatología del Alzheimer en el síndrome de Down suele iniciarse en el lóbulo temporal y en la formación hipocampal, especialmente en la corteza entorrinal, el giro dentado, la región CA1 del hipocampo, y el subículum (Millan Sanchez, Heyn, Das, Moghadam, Martin \& Salehi, 2012; Zigman \& Lott, 2007). Posteriormente, esta neuropatología tiende a extenderse al cerebelo y al mesencéfalo.

Los estudios epidemiológicos muestran, en definitiva, que es más probable desarrollar la enfermedad de Alzheimer en sujetos con síndrome de Down (Nieuwenhuis-Mark, 2009). Sin embargo, no todos los casos de síndrome de Down presentan signos de demencia, incluso aunque en ellos existan procesos neuropatológicos similares a los hallados en el Alzheimer (Fillat et al., 2010). La explicación, como se ha venido apuntando, puede residir en el hecho de que tales procesos, aun estando relacionados, no son idénticos. Puesto que la presencia de neuropatología de tipo Alzheimer no se asocia con demencia en todos los casos de síndrome de Down, la formación de placas seniles no es un indicador patológico objetivo para discriminar en estos casos una evolución hacia la demencia tipo Alzheimer (Head, Lott, Patterson, Doran \& Haier, 2007).

El porcentaje de individuos con síndrome de Down y demencia aumenta progresivamente conforme avanza la edad (Evenhuis, 1990). A partir de los cincuenta y los sesenta años el porcentaje es significativamente mayor, si bien el riesgo de estos sujetos 
de padecer demencia nunca parece alcanzar el 100\%. La proliferación de placas seniles demostrada en el síndrome de Down, desde la adultez temprana, se ha asociado más bien con un envejecimiento cognitivo precoz, que no se considera cualitativa ni cuantitativamente una demencia (Nieuwenhuis-Mark, 2009). Los estudios moleculares han demostrado que una forma específica de proteína amiloide de las placas seniles, la forma insoluble, está correlacionada con los déficit cognitivos de la enfermedad de Alzheimer (Bertram \& Tanzi, 2012). Esta proteína anómala forma cuerpos de fibrilos, y las placas seniles resultantes son, pues, fibrilares. A tales placas se les suele llamar también placas neuríticas, y son características de la demencia de Alzheimer. En cambio, estas placas fibrilares raramente se encuentran en los sujetos con síndrome de Down antes de los cincuenta años. Por lo general, las placas de $\beta$-amiloide que se forman a edades tempranas en el síndrome de Down son no fibrilares (Jones, Hanney, Francis \& Ballard, 2009). Se ha demostrado que las placas neuríticas del Alzheimer, que son de forma fibrilar, están asociadas con procesos inflamatorios y neurodegenerativos (Filippi, Canu \& Agosta, 2012). La neurodegeneración que induce estas placas, junto con los ovillos neurofibrilares, parece ser la responsable de los signos de demencia clínica en los sujetos con Alzheimer (Filippi et al., 2012). Estas placas no se observan habitualmente antes de los cuarenta años de edad, pero aumentan significativamente a partir de los cincuenta. En la edad adolescente de los sujetos con síndrome de Down también pueden observarse placas seniles, pero en esos casos las placas no parecen ser fibrilares (Lott, 2012). Algunas investigaciones han sugerido que el origen celular de ambas placas $\beta$-amiloides es diferente. Se ha apuntado que las placas fibrilares no derivan de las propias neuronas, sino de células vasculares y gliales (Mrak \& Griffin, 2005). Cuando se acumulan estas, provocan procesos patológicos extracelulares que acaban dañando a las neuronas circundantes. Estas placas y la formación de ovillos neurofibrilares se consideran la etiopatogenia de la demencia de Alzheimer (Filippi et al., 2012). La conexión entre placas seniles y síndrome de Down tiene, pues, una explicación genética (Fillat et al., 2010; Lott, 2012). El gen que codifica la proteína precursora de la $\beta$-amiloide aparece triplicado en el síndrome de Down. Pero la trisomía del par cromosómico 21 no tiene por qué implicar necesariamente la misma mutación del gen que sintetiza a esta proteína precursora que se observa en la enfermedad de Alzheimer. Por el contrario, la trisomía 21 sí parece estar relaciona con la aparición de placas proteicas difusas tempranas (Zigman \& Lott, 2007). En cualquiera de los casos, la mayor probabilidad de manifestar signos de demencia en los sujetos con síndrome de Down puede deberse al hecho de que el mismo gen alterado en la enfermedad de Alzheimer (responsable de la formación de APO E) también puede hallarse en el síndrome de Down, máxime cuando estos individuos disponen de un cromosoma 21 extra. En tales casos, la trisomía puede llevar consigo, además, una mutación del gen de la apolipoproteína, similar a la que ocurre en el Alzheimer. Esta mutación sería determinante en la expresión de demencia precoz observada en un número considerable de adultos jóvenes con síndrome de Down (Lott, 2012; Patterson, 2009). De igual modo, los ovillos neurofibrilares, que son un signo patológico prácticamente exclusivo de la demencia de Alzheimer, también tienen su origen en algún tipo de mutación genética situada, al menos, en el par cromosómico 21 (Filippi et al., 2012). La proteína tau que descompone los microtúbulos de las neuronas y forma ovillos neurofibrilares parece sintetizarse igualmente a partir de genes localizados en los cromosomas del par 21. Es posible que en sujetos con síndrome de Down y con ovillos neurofibrilares de aparición temprana (en torno a los 35 años), la trisomía afecte asimismo a los genes que sintetizan proteína tau (Jones, MargalloLana, Prasher, Ballard, 2008; Patterson, 2009). Aunque no ocurra en todos los casos, la trisomía del síndrome de Down puede elevar la probabilidad de estas otras mutaciones y, en consecuencia, puede desencadenar la aparición de demencia precoz.

\section{Discusión}

Las elaboraciones teóricas de Piaget y Vygotsky nos han ayudado a entender los complejos procesos psicológicos que tienen lugar durante el desarrollo ontogenético. Los conceptos de razonamiento, abstracción y juicios morales de la teoría piagetiana, así como la consideración del pensamiento y la palabra como una unidad en los planteamientos de Vygotsky, han tenido notables implicaciones en las teorías del aprendizaje (Daurignac, Houdé \& Jouvent, 2006; Qayumi, 2001) y en el tratamiento de algunos trastornos del desarrollo que afectan a las capacidades cognitivas (Torres, Olivares, Rodríguez, Vaamonde \& Berríos, 2007). Pero es importante destacar que los mecanismos y procesos psicológicos propuestos por estos autores tienen un sustrato neurobiológico que la Neurociencia está tratando de dilucidar. 
Uno de los trastornos del desarrollo en el que pueden conjugarse los supuestos teóricos de estos autores y los conocimientos de la Neurociencia sobre los sustratos biológicos evolutivos es el síndrome de Down. Este síndrome es una de las alteraciones del neurodesarrollo que evidencia cómo las influencias genéticas y epigenéticas condicionan el desarrollo neurobiológico y funcional del ser humano. El curso evolutivo de un individuo, desde el nacimiento hasta la vejez, depende de la integridad del sistema nervioso. Y, tal y como hemos comprobado, la formación de éste es un proceso complejo con numerosas etapas susceptibles de múltiples influencias. Las primeras etapas prenatales son críticas para el correcto desarrollo del sistema nervioso. Los factores genéticos y epigenéticos tienen una incidencia extrema sobre el neurodesarrollo, a veces con consecuencias fatales. No obstante, las etapas prenatales posteriores y los primeros años de vida son igualmente momentos determinantes para la correcta evolución del individuo. Las limitaciones genéticas y epigenéticas sobre el neurodesarrollo no son por sí mismas uniformes, y en el mismo curso evolutivo pueden converger otras condiciones suficientes para optimizar el propio desarrollo. El síndrome de Down es, de este modo, un trastorno del neurodesarrollo que limita la correcta formación del sistema nervioso y que condiciona numerosos aspectos funcionales (Sturgeon, Le, Ahmed \& Gardiner, 2012). Sin embargo, la propia plasticidad cerebral, que aunque disminuida también se manifiesta en el síndrome de Down (Baroncelli, Braschi, Spolidoro, Begenisic, Maffei \& Sale, 2011), permite que muchos de estos sujetos optimicen sus recursos más de lo que se había pensado durante muchos años. En efecto, las alteraciones en la formación y maduración del sistema nervioso que tienen lugar en el síndrome de Down, limitan el rendimiento cognitivo en general, y afectan potencialmente a procesos de aprendizaje como los descritos por Piaget, Vygotsky u otros teóricos (Jacola, Byars, Chalfonte-Evans, Schmithorst, Hickey, Patterson et al. 2011). Pero la variabilidad en las capacidades intelectuales entre los sujetos con síndrome de Down se debe al grado de afectación del sistema nervioso y a la capacidad plástica de sus cerebros (Battaglia, Quartarone, Rizzo, Ghilardi, Di Rocco, Tortorella et al. 2008). De este modo, la manifestación de tal síndrome no supone una incapacidad para determinados aprendizajes, ni impide necesariamente un adecuado desempeño cognitivo. Algunos procesos implícitos en las teorías de aprendizaje y desarrollo de Piaget o Vygotsky pueden aun manifestarse en niños con retraso mental o con síndrome de Down (Wohlhueter \& Sindberg, 1975).

Así pues, el efecto de una trisomía sobre el neurodesarrollo es inevitable, pero sus consecuencias funcionales sí pueden ser minimizadas. Consecuentemente, la relación entre neurodesarrollo y desarrollo vital es compleja pero no inflexible, y conviene recordar esto en cualquier ámbito de la Psicología, especialmente en la Psicología del Desarrollo.

\section{Referencias}

Bartesaghi, R., Guidi, S. \& Ciani, E. (2011). Is it possible to improve neurodevelopmental abnormalities in Down syndrome? Reviews in the Neurosciences, 22(4), 419-455.

Battaglia, F., Quartarone, A., Rizzo, V., Ghilardi, M.F., Di Rocco, A., Tortorella, G., Girlanda, P. (2008). Early impairment of synaptic plasticity in patients with Down's syndrome. Neurobiology of Aging, 29(8), 1272-1275.

Beal, M.F. (2000). Energetics in the pathogenesis of neurodegenerative diseases. Trends in Neuroscience, 23, 298-304.

Bertram, L. \& Tanzi, R.E. (2012). The genetics of Alzheimer's disease. Progress in molecular biology and translational science, 107, 79-100.

Carlson, N.R. (2010). Fundamentos de fisiología de la conducta. $10^{\mathrm{a}}$ Ed. Madrid: Pearson Addison-Wesley.

Changeux, J-P. (1994). Química de las comunicaciones cerebrales. Investigación y Ciencia, 208, 18-26.

Copp, A.J. (2005). Neurulation in the cranial region-normal and abnormal. Journal of Anatomy, 207(5), 623-635.

Copp, A.J. \& Greene, N.D. (2010). Genetics and development of neural tube defects. The Journal of Pathology, 220(2), 217-230.

Coppedè, F. (2009). The complex relationship between folate/homocysteine metabolism and risk of Down syndrome. Mutation research, 682, 54-70.

Daurignac, E., Houdé, O. \& Jouvent, R. (2006). Negative priming in a numerical Piaget-like task as evidenced by ERP. Journal of Cognitive Neuroscience, 18(5), 730-736.

De Marco, P., Merello, E., Cama, A., Kibar, Z. \& Capra, V. (2011). Human neural tube defects: genetic causes and prevention. Biofactors, 37(4), 261-268.

Del abril Alonso, A., Ambrosio Flores, E., De blas Calleja, M.R., Caminero Gómez, A.A., García Lecumberri, C., De pablo González, J.M. \& Sandoval Valdemoro, E. (2001). Fundamentos biológicos de la conducta. $2^{\mathrm{a}}$ Ed. Madrid: Sanz y Torres.

Diamond, M.C., Scheibel, A.B. \& Elson, L.M. (1997). El cerebro humano. Libro de trabajo. Barcelona: Ariel.

Dierssen, M., Herault, Y. \& Estivill, X. (2009). Aneuploidy: from a physiological mechanism of variance to Down syndrome. Physiological Reviews, 89(3), 887-920.

Evenhuis, H.M. (1990). The natural history of dementia in Down's syndrome. Archives of Neurology, 47, 263-267.

Farel P.B. (2003). Late differentiation contributes to the apparent increase in sensory neuron number in juvenile rat. Developmental Brain Research, 144, 91-98.

Filippi, M., Canu, E. \& Agosta F. (2012). The Role of Amyloid- $\beta$, tau, and Apolipoprotein E \{varepsilon\} 4 in Alzheimer Disease: How Is the Team Playing?. American Journal of Neuroradiology, 34(5), 511-512. DOI: 10.3174/ajnr.A3295 
Fillat, C., Dierssen, M., de Lagrán, M.M. \& Altafaj, X. (2010). Insights from mouse models to understand neurodegeneration in Down syndrome. CNS Neurol Disord Drug Targets, 9(4), 429-438.

Frieden, C. \& Garai, K. (2012). Structural differences between apoE3 and apoE4 may be useful in developing therapeutic agents for Alzheimer's disease. Proceedings of the National Academy of Sciences of the United States of America, 109(23), 8913-8918.

Galaburda A.M. \& Cestnick, L (2003). Dislexia del desarrollo. Revista de Neurología, 36, 3-9.

Gardiner, K. (2003). Predicting pathway perturbations in Down syndrome. Journal of Neural Transmission, Supplement, 67, 21-37.

Gardiner, K., Herault, Y., Lott, I.T., Antonarakis, S.E., Reeves, R.H. \& Dierssen, M. (2010). Down syndrome: from understanding the neurobiology to therapy. The Journal of Neuroscience, 30(45), 14943-14945.

Garner, C.C. \& Wetmore, D.Z. (2012). Synaptic pathology of Down syndrome. Advances in Experimental Medicine and Biology, 970, 451-468.

Gat-Yablonski, G. (2011). Brain development is a multi-level regulated process-the case of the OTX2 gene. Pediatric Endocrinology Reviews, 9(1), 422-430.

Goldberg, E. (2004). El cerebro ejecutivo: lóbulos frontales y mente civilizada. Barcelona: Crítica.

Greene, N.C. \& Copp, A. J. (2009). Development of the vertebrate central nervous system: formation of the neural tube. Prenatal Diagnosis, 29(4), 303-311

Harvey L. \& Boksa, P. (2012). Prenatal and postnatal animal models of immune activation: Relevance to a range of neurodevelopmental disorders: Developmental Neurobiology Special Issue: Neuroimmunology in Development and Disease. Developmental Neurobiology, 72(10), 1335-1348. DOI: 10.1002/dneu.22043

Head, E., Lott, I.T., Patterson, D., Doran, E. \& Haier, R.J. (2007). Possible compensatory events in adult Down syndrome brain prior to the development of Alzheimer disease neuropathology: targets for nonpharmacological intervention. Journal of Alzheimer's Disease, 11(1), 61-76.

Hernández-Muela, S., Mulas, F. \& Mattos, L. (2004). Plasticidad neuronal funcional. Revista de Neurología, 38(1), 58-68.

Hsiao, E.Y. \& Patterson, P.H. (2012). Placental regulation of maternal-fetal interactions and brain development. Developmental Neurobiology, 72(10), 1317-1326. DOI: 10.1002/dneu.22045.

Jacola, L.M., Byars, A.W., Chalfonte-Evans, M., Schmithorst, V.J., Hickey, F., Patterson, B., Hotze, S., Vannest, J., Chiu, C.Y., Holland, S.K. \& Schapiro, M.B. (2011). Functional magnetic resonance imaging of cognitive processing in young adults with Down syndrome. American Journal on Intellectual and Developmental Disabilities, 116(5), 344-359.

Jones, E.L., Hanney, M., Francis, P.T. \& Ballard, C.G. (2009). Amyloid beta concentrations in older people with Down syndrome and dementia. Neuroscience Letters, 451(2), 162-164.

Jones, E.L., Margallo-Lana, M., Prasher, V.P. \& Ballard, C.G. (2008). The extended tau haplotype and the age of onset of dementia in Down syndrome. Dementia and Geriatric Cognitive Disorders, 26(3), 199-202.

Kalat, J.W. (2004). Psicología biológica. Madrid: Thompson.

Kandel, E.R., Jessell, T.M. \& Schwartz, J.H (2003). Neurociencia y conducta. Madrid: Prentice Hall.

Kleschevnikov, A.M., Belichenko, P.V., Salehi. A. \& Wu, C. (2012). Discoveries in Down syndrome: moving basic science to clinical care. Progress in Brain Research, 197, 199-221.

Le vay (1995). El cerebro sexual. Madrid: Alianza.

Lott, I.T. (2012). Neurological phenotypes for Down syndrome across the life span. Progress in Brain Research, 197, 101-121.
Lott, I.T. \& Dierssen, M. (2010). Cognitive deficits and associated neurological complications in individuals with Down's syndrome. The Lancet Neurology, 9(6), 623-633.

Mardomingo, M.J. (1997). Psicofarmacología del niño y del adolescente. Madrid: Díaz de Santos.

Millan Sanchez, M., Heyn, S.N., Das, D., Moghadam, S., Martin, K.J. \& Salehi, A. (2012). Neurobiological elements of cognitive dysfunction in down syndrome: exploring the role of APP. Biological Psychiatry, 71(5), 403-409.

Molero, A., Velo, M.S. y Lorca, J.A. (2007). Características clínicas, neuropatológicas y sociales de la enfermedad de Alzheimer y otras demencias. La memoria, 17, 5-9.

Mrak, R.E. \& Griffin, W.S. (2005). Glia and their cytokines in progression of neurodegeneration. Neurobiology of Aging, 26(3), 349-354.

Nelson, R.J. (1996). Psicoendocrinología: las bases hormonales de la conducta. Barcelona: Ariel.

Nickl-Jockschat, T. \& Michel, T.M. (2011). The role of neurotrophic factors in autism. Molecular Psychiatry, 16(5), 478-490.

Nieuwenhuis-Mark, R.E. (2009). Diagnosing Alzheimer's dementia in Down syndrome: problems and possible solutions. Research in Developmental Disabilities, 30(5), 827-838.

Parajuá-Pozo, J.L. \& Casis-Arguea, S. (2000). Síndrome de Down y demencia. Revista de Neurología, 31, 126-128.

Parellada, E. (2001). Neurodesarrollo frente a neurodegeneración: hipótesis neuroanatómicas de la esquizofrenia. Psiquiatría Biológica, 6, 225-230.

Patterson, D. (2009). Molecular genetic analysis of Down syndrome. Human genetics, 126, 195-214.

Perluigi, M. \& Butterfield, D.A. (2012). Oxidative Stress and Down Syndrome: A Route toward Alzheimer-Like Dementia. Current Gerontology and Geriatrics Research, in press.

Piaget, J. (1977). La naissance de l'intelligence chez l'enfant. Delachaux \& Niestlé, S.A., Neuchâtel.

Piaget, J. (1985). El nacimiento de la inteligencia en el niño. Barcelona: Crítica, D.L.

Piaget, J. \& Inhelder, B. (1969). La psychologie de l'enfant. Paris: Presses Universitaires de France.

Piaget, J. \& Inhelder, B. (1993). Psicología del niño. $13^{\text {a }}$ Ed. Madrid: Morata.

Pinel, J.P.J. (2001). Biopsicología. $4^{a}$ Ed. Madrid: Prentice Hall.

Pinos Sánchez, H., Collado Guirao, P. \& Sánchez-Santed, F. (2005). Apoptosis y neurogénesis: dos procesos implicados en el desarrollo del dimorfismo sexual del sistema nervioso. España: Publicaciones UNED

Plomin, R. (2002). Genética de la conducta. $4^{\mathrm{a}}$ Ed. Barcelona: Ariel.

Preatoni, A. (2003). Anatomía del desarrollo del sistema nervioso. Santa Fe: El Cid Editor.

Puelles, L., Martínez, S. \& Martínez, M. (2008). Neuroanatomía. Madrid: Editorial Médica Panamericana.

Qayumi, S. (2001). Piaget and his role in problem based learning. Journal of Investigative Surgery, 14(2), 63-65.

Ramon y Cajal, S. (1904). Textura del sistema nervioso del hombre y los vertebrados. Tomo II. (309-448). Madrid: N. Moya

Rosenzweig, M.R. \& Leiman, A.L. (2002). Psicología biológica. Madrid: McGraw Hill.

Sabbagh, M.N., Fleisher, A., Chen, K., Rogers, J., Berk, C., Reiman, E., Pontecorvo, M., Mintun, M., Skovronsky, D., Jacobson. S.A., Sue, L.I., Liebsack, C., Charney, A.S., Cole, L., Belden, C., Beach, T.G. (2011). Positron emission tomography and neuropathologic estimates of fibrillar amyloid- $\beta$ in a patient with Down syndrome and Alzheimer disease. Archives of Neurology, 68(11), 1461-1466.

Sadler, T.W. (2005). Embryology of neural tube development. American Journal of Medical Genetics Part C: Seminars in Medical Genetics, 135(1), 2-8. 
Sanes, D.H., Reh, T.A. \& Harris, W.A. (2002). El desarrollo del sistema nervioso. Barcelona: Ariel Neurociencia.

Scott, G.J., Holding, S., Purcell, A., Tutty, S. \& Lindow, S.W. (2009). The influence of maternal opiate use in pregnancy on second trimester biochemical markers for Down syndrome. Prenatal diagnosis, 29, 863-865.

Segovia, S. \& Guillamón, S. (Eds.) (1988). Psicobiología del desarrollo. Barcelona: Ariel.

Shi, Y., Kirwan, P., Smith, J., MacLean, G., Orkin, S.H. \& Livesey, F.J. (2012). A human stem cell model of early Alzheimer's disease pathology in Down syndrome. Science Translational Medicine, 4(124), 124-129.

Snell, S. (1999). Neuroanatomía clínica. Madrid: Panamericana.

Sommer, C.A. \& Henrique-Silva, F. (2008). Trisomy 21 and Down syndrome. A short review. Brazilian Journal of Biology, 68, 447-452.

Stansfield, W.D. (1992). Genética. $3^{\text {a }}$ Ed. Madrid: McGraw Hill.

Stiles, J. \& Jernigan, T.L. (2010). The basics of brain development. Neuropsychology Review, 20(4), 327-348.
Sturgeon, X., Le, T., Ahmed, M.M. \& Gardiner, K.J. (2012). Pathways to cognitive deficits in Down syndrome. Progress in Brain Research, 197, 73-100.

Torres, A., Olivares, J.M., Rodriguez, A., Vaamonde, A. \& Berrios, G.E. (2007). An analysis of the cognitive deficit of schizophrenia based on the Piaget developmental theory. Comprehensive Psychiatry, 48(4), 376-379.

Vygotsky, L. (1993). Pensamiento y lenguaje. Teoría del desarrollo cultural de las funciones psíquicas. Buenos Aires: Fausto.

Wiseman, F.K., Alford, K.A., Tybulewicz, V.L.J. \& Fisher, E.M.C. (2009). Down síndrome-recent progress and future prospects. Human Molecular Genetics, 18, 75-83.

Wohlhueter, M.J. \& Sindberg, R.M. (1975). Longitudinal development of object permanence in mentally retarded children: an exploratory study. American Journal of Mental Deficiency, 79(5), 513-518.

Zigman, W.B., Lott, I.T. (2007). Alzheimer's disease in Down syndrome: neurobiology and risk. Mental Retardation and Developmental Disabilities Research Reviews, 13(3), 237-246. 